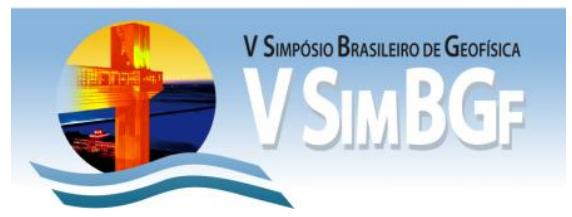

\title{
Mapeamento da distribuição de fluidos em um modelo de reservatório pinch-out utilizando modelagem física e atributos sísmicos instantâneos
}

Carlos A. M. de Assis* - carlosmartinsx@hotmail.com

Marco A. R. de Ceia, Wagner M. Lupinacci e Roseane M. Misságia

LENEP/UENF

\section{Copyright 2012, SBGf - Sociedade Brasileira de Geofísica}

Este texto foi preparado para a apresentação no V Simpósio Brasileiro de Geofísica Salvador, 27 a 29 de novembro de 2012. Seu conteúdo foi revisado pelo Comitê Técnico do V SimBGf, mas não necessariamente representa a opinião da SBGf ou de seus associados. É proibida a reprodução total ou parcial deste material para propósitos comerciais sem prévia autorização da SBGf.

\begin{abstract}
This work aims to show the results obtained from experiments using seismic physical modeling techniques to image non-homogeneous, two phase distribution of immiscible fluids inside a cavity on a pinch-out model. The main goal is to verify how the seismic sections can be used to observe the fluid distribution in this type of reservoir. A robust deconvolution method was applied to improve resolution and instantaneous attributes were used to help the data interpretation. The results provided an image of the oil-water interface, which reveal a complex fluid compartmentalization pattern. We hope that those studies can improve the understanding in mapping fluid distribution from seismic sections on pinch-out reservoirs.
\end{abstract}

\section{Introdução}

A tecnologia sísmica tem sido utilizada na exploração de hidrocarbonetos por mais de meio século. Inicialmente apenas linhas sísmicas 2D eram adquiridas. Então, a partir de 1970 os levantamentos 3D começaram a ser usados não apenas para exploração, mas também para a caracterização de reservatórios e com isso têm auxiliado no desenvolvimento de campos de óleo e gás (Grochau, 2009). A sísmica de exploração normalmente tem como foco o mapeamento das estruturas em subsuperfície. Com a sua aplicação no monitoramento de reservatórios - foco passa a ser as mudanças na saturação e distribuição dos fluidos, nos gradientes de pressão e na temperatura do reservatório. Com o melhor entendimento da subsuperfície é possível diminuir as incertezas associadas aos dados dos simuladores de fluxo e aperfeiçoar o posicionamento de poços (injetores e produtores), como relatado em estudos de caso por Gouveia et al., (2004) e Gonzalez-Carballo et al., (2006). O método sísmico de reflexão pode ser estudado e aprimorado com dados experimentais obtidos na modelagem física sísmica. Neste tipo de modelagem utiliza-se um modelo geológico em escala de laboratório, construído comumente utilizando-se materiais como metal ou plástico. Uma fonte, normalmente um transdutor piezoelétrico, emite energia sísmica e os campos de onda refletidos no modelo são registrados dando origem a um sismograma. Existem muitos trabalhos que utilizam este tipo de modelagem com o objetivo de testar algoritmos de processamento sísmico, melhorar técnicas de aquisição e observar a resposta sísmica que um determinado modelo produz. Mu \& Cao (2004) realizaram experimentos em um modelo com areia, no qual se variou a saturação dos fluidos $\left(\mathrm{CH}_{4}, \mathrm{CO}_{2}\right.$, óleo e água) e foi estudada a resposta sísmica em função do fator de absorção. Wang et al., (2009) estudaram os padrões gerados em sismogramas por uma caixa de areia saturada por gás, óleo e água. Cooper et al., (2010) estudaram a resposta sísmica que um modelo na forma de cunha produz (difração, múltiplas e conversões de modo) nos sismogramas, utilizando dados sintéticos para apoiar a interpretação dos dados experimentais. Sherlock et al., (2000) simularam a sísmica 4D em caixas de areia, variando a saturação dos fluidos no modelo e obtiveram sucesso no mapeamento dos fluidos nos sismogramas. Além disso, Sherlock et al., (2000) também realizaram um experimento com um modelo preenchido por gás e água, no qual foi possível mapear o contato entre estes fluidos com 0 atributo sísmico cosseno da fase instantânea. Os atributos sísmicos algumas vezes ajudam a visualizar feições, relações e padrões que de outra forma talvez não fossem percebidas (Sheriff, 2005). Neste trabalho, realizamos medidas sísmicas ultrassônicas (escala de laboratório) em um modelo composto por um bloco de plexiglass com uma cavidade na forma de cunha que permite ser preenchida por diferentes fluidos. Esta cavidade foi feita para simular um reservatório do tipo pinch-out ou uma trapa. Exemplos de reservatórios com geometria pinch-out são: Gulfaks (Noruega), Marlim e Peregrino (Brasil). Salmoura e óleo de máquina foram utilizados para simular água da formação e petróleo, respectivamente. A idéia principal no experimento realizado é mapear a distribuição de fluidos, utilizando atributos sísmicos (fase instantânea e amplitude instantânea), para detectar o contato óleo-água e verificar a resposta sísmica que a geometria do modelo produz.

\section{Configuração do experimento}

Este trabalho foi realizado em três etapas, são elas: aquisição sísmica, processamento e interpretação dos resultados. $O$ modelo utilizado foi construído com plexiglass na forma de um bloco $(300 \mathrm{~mm} \times 200 \mathrm{~mm} \times$ $180 \mathrm{~mm}$ ), que posteriormente foi perfurado para formar uma cavidade do tipo cunha (Figura 1). 


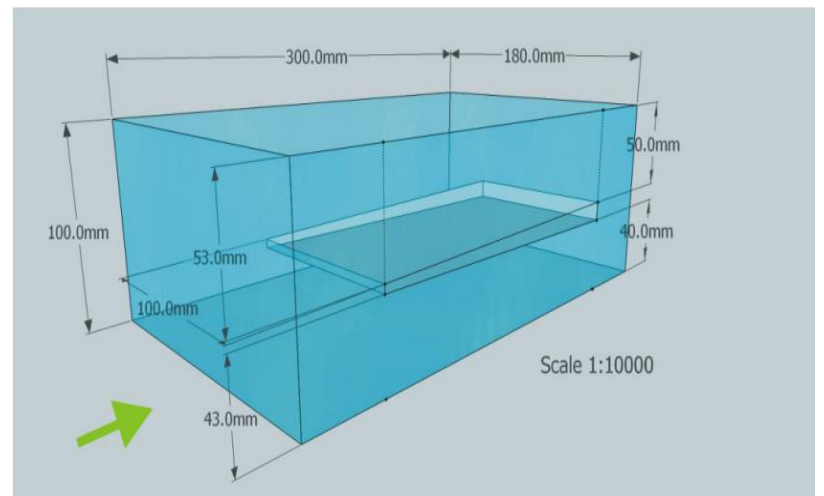

Figura 1 - llustração do modelo pinch-out. A cavidade na forma de cunha permite a acomodação de fluidos. Escala 1:10.000. A seta verde indica a direção de aquisição.

A escala de construção do modelo foi de 1:10.000. A medição direta da velocidade na parte do modelo sem a cavidade resultou em $2777 \mathrm{~m} / \mathrm{s}$ para a velocidade de onda $\mathrm{P}$ no plexiglass. Em nosso experimento apenas fluido (salmoura e óleo de máquina) foi utilizado para preencher a cavidade. A tabela 1 lista as propriedades físicas destes fluidos. Para preencher a cavidade 0 modelo foi rotacionado de forma que esta pudesse ser acessada do topo. O mesmo volume $(70 \mathrm{ml})$ de salmoura e óleo de máquina foi usado. Para selar a cavidade nós usamos um grampo $\mathrm{C}$ comprimindo uma tira de borracha e uma placa de alumínio. Silicone foi aplicado nas bordas da placa de borracha para evitar vazamentos. Devido à diferença de viscosidade dos fluidos, quando o modelo foi rotacionado novamente para a sua posição original, como mostrada na Figura 1, os fluidos assumiram uma distribuição heterogênea, como pode ser visualizado na Figura 2. Os números romanos na Figura 2 foram usados para facilitar a etapa de interpretação. I é a região apenas com água, II região com óleo e III a região com óleo na parte superior e água na parte inferior da cavidade. Então o modelo foi posicionado dentro de um tanque com água para a realização das medidas ultrassônicas. A distância da superfície da água para o topo do modelo foi mantida em $100 \mathrm{~mm}$.

Tabela 1 - Propriedades físicas dos fluidos utilizados para preencher a cavidade.

\begin{tabular}{|c|c|c|}
\hline PropriedadelFluido & Salmoura & $\begin{array}{c}\text { Óleo de } \\
\text { máquina }\end{array}$ \\
\hline Densidade $\left(\mathrm{g} / \mathrm{cm}^{3}\right)$ & 1,023 & 0,864 \\
\hline $\begin{array}{c}\text { Velocidade da } \\
\text { onda P }(\mathrm{m} / \mathrm{s})\end{array}$ & 1544 & 1384 \\
\hline $\begin{array}{c}\text { Concentração } \\
(\mathrm{NaCl})\end{array}$ & $40 \mathrm{~g} / \mathrm{l}$ & - \\
\hline
\end{tabular}

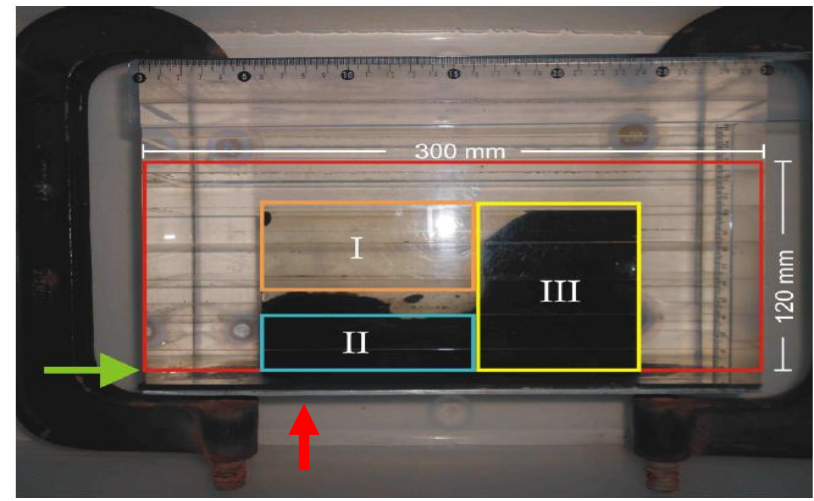

Figura 2 - Fotografia do topo do modelo pinch-out. $O$ retângulo vermelho indica a área de aquisição. A seta verde indica a direção de aquisição e o início da primeira linha. Os números romanos dividem a cavidade em regiões para a etapa de interpretação. A seta vermelha indica a direção e sentido da crossline.

\section{Aquisição dos dados}

As medidas foram realizadas no laboratório de modelagem física do Lab. de Engenharia e Exploração de Petróleo - Universidade Estadual Norte Fluminense (UENF). O equipamento de mono-canal inclui: temporizador, placas de geração de sinais e de digitalização, amplificadores de baixo ruído e transdutores de contato com freqüência central de 1 $\mathrm{MHz}$. A aquisição dos dados consistiu de 60 linhas de $300 \mathrm{~mm}$ cada ao longo do modelo pinch-out, utilizando afastamento comum. O afastamento foi fixado em 28 $\mathrm{mm}$. O sinal transmitido foi uma forma de onda tone burst janelada pela função de Bartlett. A freqüência central para o experimento foi configurada para $500 \mathrm{kHz}$ para corresponder a $50 \mathrm{~Hz}$ (escala 1:10.000), que é uma freqüência aproximada da utilizada em levantamentos sísmicos de campo. O passo entre os traços e as linhas foi de $2 \mathrm{~mm}$. A tabela 2 lista outros parâmetros utilizados para as medidas. Após a aquisição, as 60 linhas foram concatenadas em um único arquivo no formato sgy e a geometria de aquisição foi inserida em seu header.

\section{Processamento dos dados}

O processamento foi composto pela correção estática, filtragem passa banda, re-amostragem e a deconvolução dos dados. A correção estática foi realizada para corrigir pequenas variações no tempo de chegada dos eventos. A filtragem passa banda foi utilizada para remover ruídos de baixa freqüência, provavelmente originados pela agitação da água no tanque devido o movimento dos transdutores, e para preparar os dados para a reamostragem. A re-amostragem de $0,1 \mu \mathrm{s}$ para $0,25 \mu \mathrm{s}$ colaborou na diminuição do tempo de processamento na etapa de deconvolução. Por último aplicou-se um método robusto de deconvolução esparsa no domínio da freqüência (Lupinacci, 2010). Os dados foram limitados na região da cavidade para diminuir o tempo de processamento. A deconvolução eliminou reverberações que podem estar associadas a múltiplas e a oscilação dos transdutores piezoelétricos (Buddensiek et al., 2009) e aumentou a resolução dos dados. A Figura 3 mostra 0 espectrograma de um traço, calculado utilizando a 
transformada de Fourier janelada, limitado na região da cavidade, antes e depois da deconvolução. Observa-se o aumento no conteúdo de freqüência e a compressão dos eventos.

Tabela 2 - Lista de parâmetros utilizados na aquisição dos dados.

\begin{tabular}{|l|l|}
\hline $\begin{array}{l}\text { Freqüência de amostragem do sinal } \\
\text { transmitido }\end{array}$ & $4 \mathrm{MHz}$ \\
\hline Número de amostras (sinal transmitido) & 5000 \\
\hline Duração (sinal transmitido) & $1,25 \mathrm{~ms}$ \\
\hline Sinal & Tone Burst \\
\hline Função janela & Bartlett \\
\hline Freqüência do "Tone Burst" & $500 \mathrm{kHz}$ \\
\hline Fase inicial do "Tone Burst" & 0 graus \\
\hline Ciclos do "Tone Burst" & 1 \\
\hline $\begin{array}{l}\text { Número de tiros para ompilhamento } \\
\text { vertical }\end{array}$ & 100 \\
\hline $\begin{array}{l}\text { Freqüência de amostragem do sinal } \\
\text { recebido }\end{array}$ & $10 \mathrm{MHz}$ \\
\hline Número de amostras (sinal recebido) & 3000 \\
\hline Tempo de registro (sinal recebido) & $0,3 \mathrm{~ms}$ \\
\hline
\end{tabular}

Após o processamento foi gerado um cubo de dados, que foi carregado no software de interpretação sísmica Opendtect 4.2.0v (Figura 4), proporcionando além das inlines na direção de aquisição a possibilidade de se visualizar crosslines (seta vermelha na Figura 2) e timeslices (fatias de tempo constante).

\section{Atributos sísmicos instantâneos}

Um traço sísmico pode ser representado como a parte real de um sinal complexo. A partir do traço sísmico complexo é possível obter os atributos envelope de amplitude (amplitude instantânea) e fase instantânea. Esses atributos são chamados de atributos instantâneos, porque eles descrevem concisamente e quantitativamente a onda sísmica em qualquer ponto de amostra (White, 1991). Os atributos instantâneos descrevem a forma do traço sísmico e podem ser aplicados para auxiliar na interpretação dos dados. Utilizar o cosseno da fase instantânea para visualizar seções sísmicas pode ser interessante, pois este atributo atua como um ganho automático o que favorece a continuidade dos eventos (Barnes, 2007). Taner (2001) indica que a amplitude instantânea pode ser um discriminador efetivo do limite entre camadas e que esta representa principalmente o contraste de impedância acústica do meio. Neste trabalho, os atributos instantâneos cosseno da fase e amplitude são utilizados para facilitar a visualização de detalhes de interesse na etapa de interpretação. a)
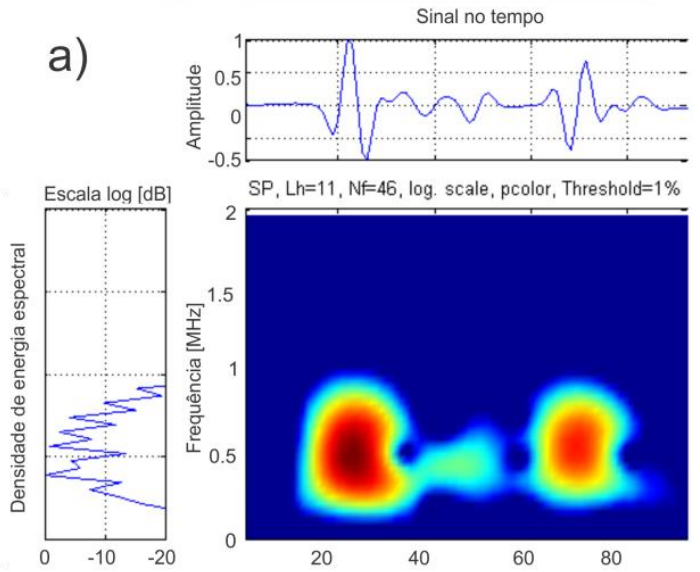

SP, Lh=11, Nf=46, log. scale, pcolor, Threshold $=1 \%$

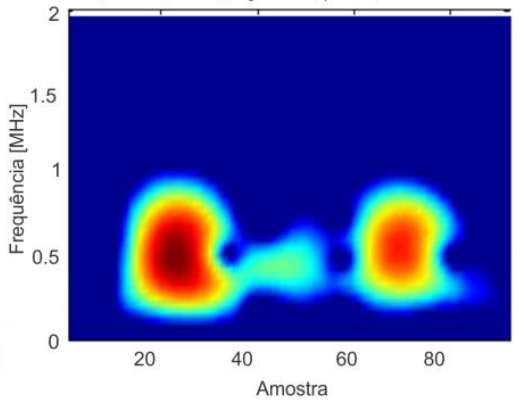

b)
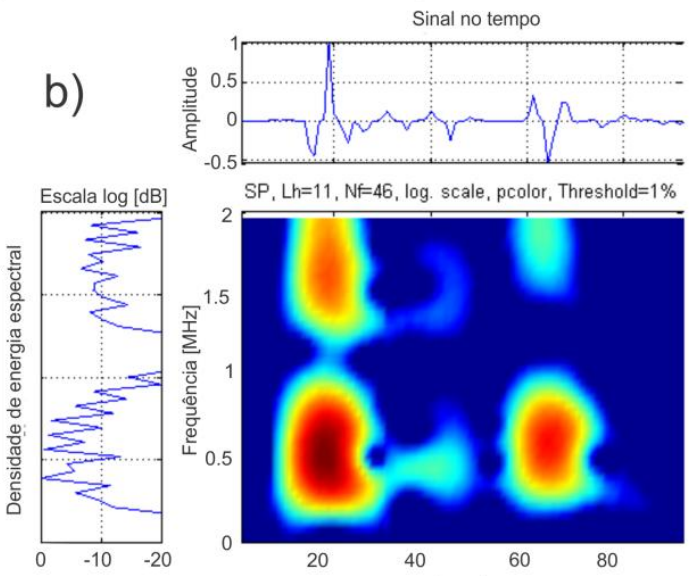

SP, Lh=11, Nf=46, log. scale, pcolor, Threshold $=1 \%$

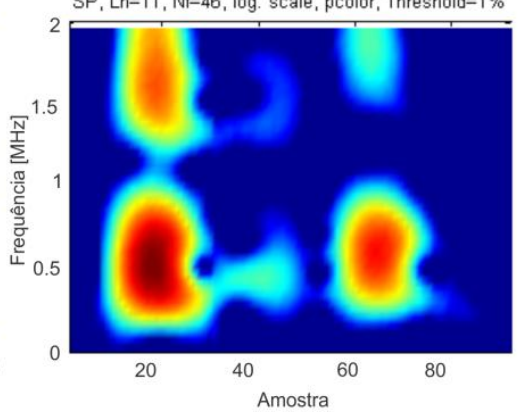

Figura 3 - Espectrograma de um traço, calculado utilizando a transformada de Fourier janelada, limitado na região da cavidade, antes (a) e depois (b) da deconvolução.

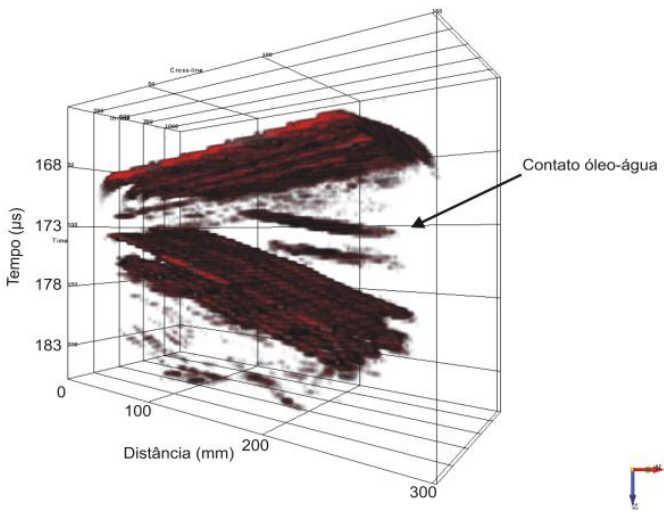

Figura 4 - Visão 3D da cavidade em função da energia. $O$ contato óleo-água pode ser visualizado claramente.

\section{Resultados}

A Figura 4 mostra o cubo de dados limitado na região da cavidade, visualizado em função da energia, do qual foram extraídas inlines, timeslices e crosslines para as interpretações. Nesta figura o contato óleo-água (região 
III) pode ser visualizado claramente. A Figura 5 mostra a linha 6, esta foi adquirida a $12 \mathrm{~mm}$ da posição inicial. Fortes amplitudes marcam as principais estruturas do modelo, como o topo do plexiglass e o topo e a base da cavidade. A base da cavidade pode ser observada como um refletor de amplitude um pouco mais fraca em relação ao topo da cavidade. Como o modelo foi posicionado sobre quatro suportes de plástico para torná-lo mais estável dentro do tanque, ocorreu outra reflexão logo abaixo da que está relacionada com a base do tanque. Uma múltipla do topo do modelo foi observada como um refletor contínuo e de forte amplitude em $270 \mu \mathrm{s}$ e ocorreram difrações nas bordas do reservatório. Para a etapa de processamento os dados foram limitados na região da cavidade, pois o objetivo principal deste trabalho é estudar a distribuição dos fluidos. Com isso também se reduziu o tempo de processamento. A Figura 6 mostra a linha 1 (região I e III da Figura 2) em função do atributo amplitude instantânea antes e depois da deconvolução. Observa-se o contato óleo-água mergulhando suavemente e o aumento da resolução (melhor definição e afinamento) dos eventos do topo e da base da cavidade e da região dentro desta. A Figura 7 mostra a crossline 80 , que atravessa as regiões I e II da Figura 2. Nesta imagem é possível visualizar o atraso no tempo de chegada da parte da base da cavidade que atravessa a região II. Isto ocorreu devido à maior velocidade de propagação das ondas refletidas na região I, preenchida com água, do que na região II que está preenchida com óleo. A Figura 8 mostra a crossline 115, que está posicionada na região III. Nesta imagem observava-se o contato óleo-água na parte central da cavidade e a base da cavidade apresenta-se retilínea devido à distribuição aproximadamente uniforme dos fluidos nesta parte do modelo. Também foi extraída uma timeslice (fatia de tempo constante paralela ao topo do modelo) dos dados, com a intenção de se observar a distribuição espacial dos fluidos. A Figura 9 mostra a timeslice $173,5 \mu$ s visualizada em função do atributo cosseno da fase instantânea. O contato óleo-água aparece aproximadamente como uma linha, devido à sua suave inclinação como visto na seção sísmica da Figura 6.

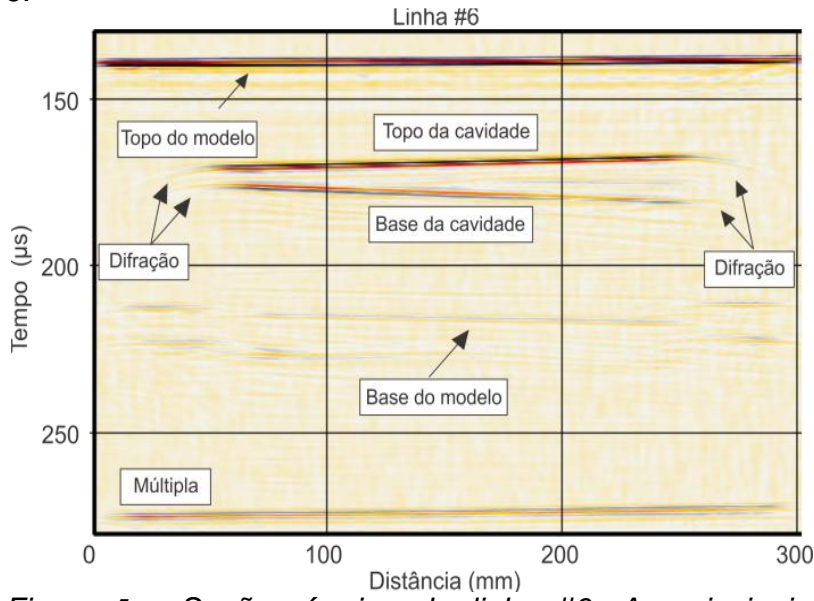

Figura 5 - Seção sísmica da linha \#6. As principais estruturas do modelo podem ser observadas no sismograma.

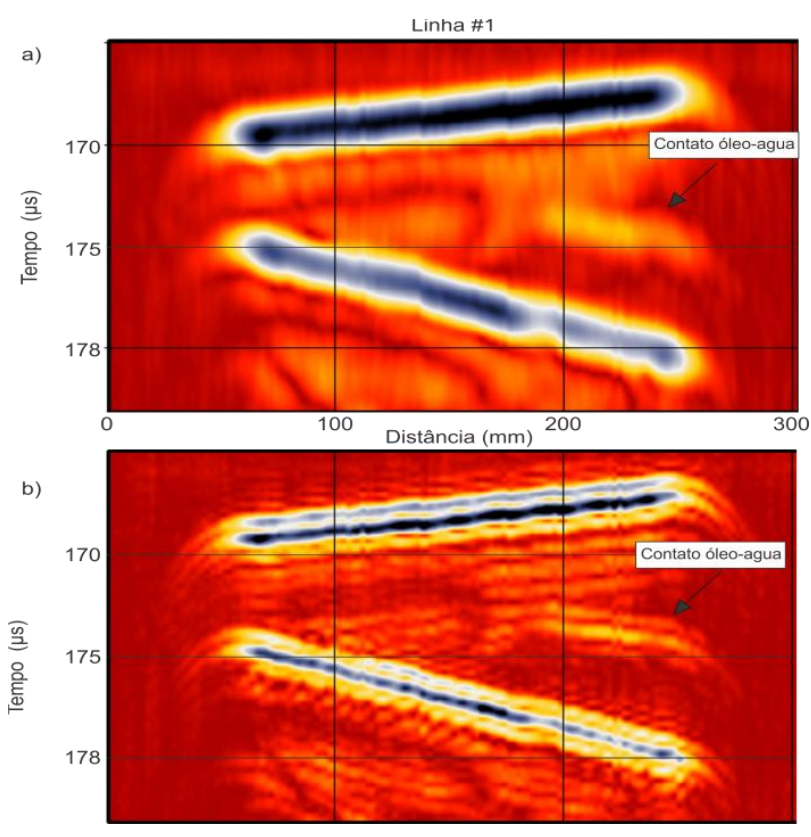

Figura 6 - Seção sísmica da linha \#1 visualizada em função do atributo amplitude instantânea. (a) Antes da deconvolução; (b) Após a deconvolução.

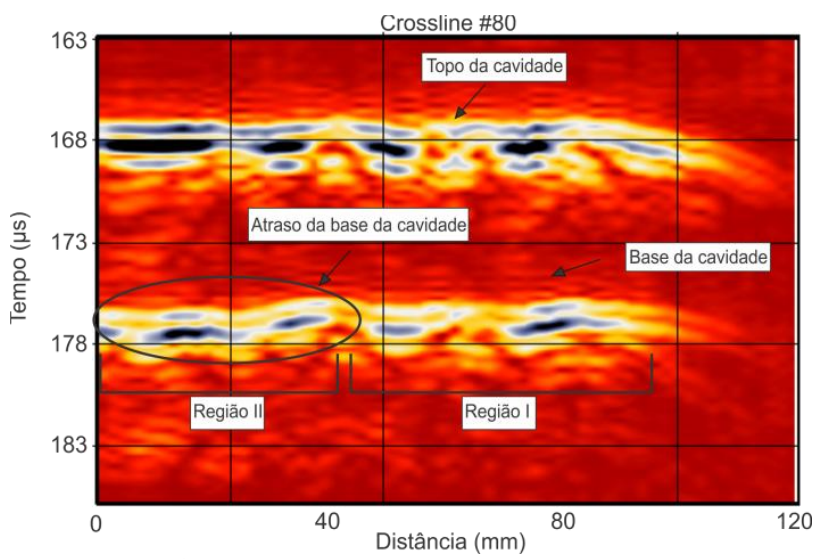

Figura 7 - Seção sísmica da crossline \#80 (atravessa regiões I e II) visualizada em função do atributo amplitude instantânea.

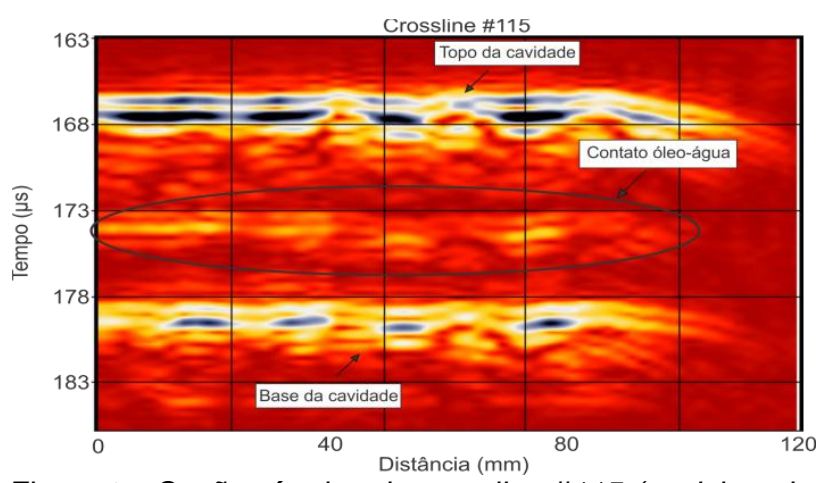

Figura 8 - Seção sísmica da crossline \#115 (posicionada na região III) visualizada em função do atributo amplitude instantânea. 


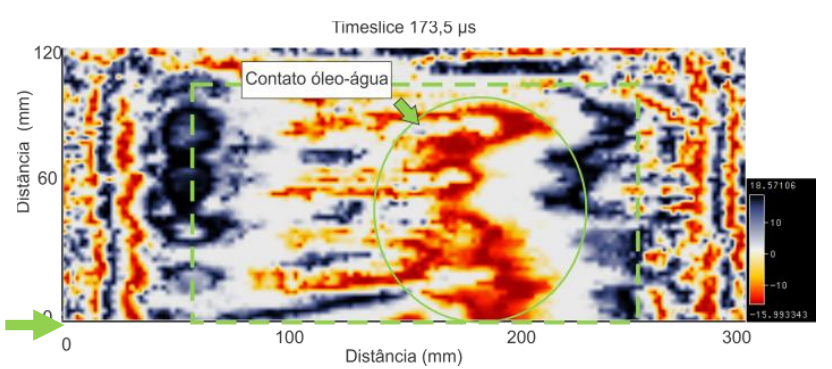

Figura 9 - Seção sísmica da timeslice no tempo 173,5 $\mu$ s, visualizada em função do atributo cosseno da fase instantânea. O retângulo tracejado indica a área do reservatório. A seta verde indica a direção de aquisição.

\section{Discussão e Conclusões}

Os experimentos realizados no modelo pinch-out para o mapeamento da distribuição de fluidos foram bem sucedidos. Três regiões diferentes da cavidade foram interpretadas, baseando-se na assinatura sísmica e em imagens do modelo. A região III está saturada por óleo e água. A água da região III invade uma parte da região II, que esta saturada principalmente com óleo. A região I está saturada apenas com água. Nos sismogramas foi possível observar o contato óleo-água (Figuras 6, 8 e 9) e o efeito de deslocamento da base do reservatório na seção sísmica (Figura 7) que atravessa a região que começa com óleo (região II) e em determinado ponto passa a ser saturada apenas por água (região I). Também foi possível testar em dados ultrassônicos um algoritmo de deconvolução de alta resolução, que foi desenvolvido para dados sísmicos reais. Este algoritmo proporcionou um aumento de resolução nas imagens o que auxiliou na interpretação dos dados. O uso dos atributos sísmicos colaborou na etapa de interpretação realçando detalhes de interesse (contato óleo-água, base e topo da cavidade), sendo possível confirmar as suas aplicações previstas na teoria. Ainda que não tenha sido simulado um meio poroso dentro da cavidade, o padrão de distribuição dos fluidos depois que o modelo foi rotacionado forneceu uma geometria interessante, resultando em uma distribuição heterogênea dos fluidos dentro do reservatório.

\section{Agradecimentos}

Agradecemos à Petrobras pelo financiamento do sistema de modelagem física. Ao Marcus Freire pelas discussões sobre o método sísmico de reflexão e aquisição dos dados. A Adrielle Silva, Remilson Rosa, Evandro Henrique e Jhonnes Silva pela colaboração durante os experimentos. E ao PRH20-ANP pela bolsa de iniciação científica.

\section{Referências}

Barnes, A.E., 2007. A tutorial on complex seismic trace analysis. Geophysics, Society of Exploration Geophysicists, 72(6), W33-W43.

Buddensiek, M.; Krawczyk, C.; Kukowski, N.; Oncken, O., 2009. Performance of piezoelectric transducers in terms of amplitude and waveform. Geophysics, Society of Exploration Geophysicists, 74(2), T33-T45.

Cooper, J.; Lawtown, D.; Margrave, G., 2010. The wedge model revisited: A physical modeling experiment. Geophysics, Society of Exploration Geophysicists, 75(2), T15-T21.

Gonzalez-Carballo, A.; Guyonnet, P.; Levallois, B.; Veillerette, A.; Deboiasne, R., 2006. 4D monitoring in Angola and its impact on reservoir understanding and economics. The Leading Edge, Society of Exploration Geophysicists, 25(9), 1150-1159.

Gouveia, W.; Johnston, D.; Solberg, A.; Lauritzen, M., 2004. Jotun 4D: Characterization of fluid contact movement from time-lapse seismic and production logging tool data. The Leading Edge, Society of Exploration Geophysicists, 23, 1187.

Grochau, Marcos Hexsel, 2009. An Integrated approach to improve time-lapse seismic interpretation: Investigation of pressure and saturation effects on elastic parameters. Saarbrücken: Lambert Academic Publishing.

Lupinacci, W. M., 2010. Um método robusto de deconvolução esparsa no domínio da freqüência. Macaé: Universidade Estadual do Norte Fluminense Darcy Ribeiro / Laboratório de Engenharia e Exploração de Petróleo.

Mu, Y.; Cao, S., 2004. Seismic physical modeling and sandstone reservoir detection using absorption coefficients of seismic reflections. Journal of Petroleum Science and Engineering, Elsevier, 41(1), 159-167.

Sheriff, Robert E., 2005. Encyclopedic dictionary of exploration geophysics. 4.ed. Society of Exploration Geophysicists.

Sherlock, D.; Mckenna, J.; Evans, B., 2000. Time-lapse 3D seismic physical modeling. Exploration Geophysics, CSIRO, 31(2), 310-314.

Taner, M.T, 2001. Seismic attributes. CSEG recorder, 26(7), 48-56.

Wang, S.; DI, B.; Wei, J.; Zhang, S., 2009 Physical modeling experiment and analysis of seismic response of a reservoir model filled with different fluids. In: 2009 SEG Annual Meeting.

White, R. E., 1991. Properties of instantaneous seismic attributes. The Leading Edge, Society of Exploration Geophysicists, 10(7), 26-32. 